\title{
ROBUST VEHICLE STEERING CONTROL DESIGN BASED ON THE DISTURBANCE OBSERVER
}

\author{
Tilman Bünte * Dirk Odenthal * Bilin Aksun-Güvenç ** \\ Levent Güvenç ${ }^{* *, 1}$ \\ * DLR, German Aerospace Center, \\ Institute of Robotics and Mechatronics, \\ Oberpfaffenhofen, D-82230 Wessling, Germany \\ Fax: +49-8153-281847 e-mail: Tilman.Buente@dlr.de \\ ** Istanbul Technical University \\ Dept. of Mech. Eng., Gümü̧ssuyu, Taksim, Istanbul, Turkey
}

\begin{abstract}
A robust steering controller is introduced for improving the yaw dynamics of a passenger car. A specific two degree of freedom control architecture known as the disturbance observer is adapted to the vehicle yaw dynamics control problem and shown to robustly improve performance. The relevant design specifications are formulated in terms of eigenvalues ( $\Gamma$-stability) and in frequency domain as bounds on weighted sensitivity and complementary sensitivity functions ( $\mathcal{B}$-stability). The parameter space method is used to map the specifications for controller design. A Popov criterion based nonlinear stability analysis is also carried out to prove robust absolute stability ( $\Theta$-stability) in the presence of actuator rate limitation. Simulations are used to demonstrate the effectiveness of the final design.
\end{abstract}

Keywords: Vehicle Dynamics Control, Disturbance Observer, Parameter Space Method, Robust Control.

\section{INTRODUCTION}

Dangerous yaw motions of an automobile may result from unexpected yaw disturbances caused by unsymmetrical car dynamics perturbations like unilateral loss of tire pressure or braking on unilaterally icy road ( $\mu$-split braking). Safe driving requires the driver to react extremely quickly in such dangerous situations. This is not possible as the driver who can be modeled as a high gain control system with dead time overreacts, resulting in instability. Consequently, improvement of automobile yaw dynamics by active control to avoid such catastrophic situations has been and is continuing to be a subject of active research. In this paper, a steer-by-wire system is assumed

\footnotetext{
1 acknowledges the support of the Alexander von Humboldt Stiftung
}

where the commands to the steering actuator from the steering controller are added to those of the driver commanded via the steering wheel. The steering control system should be robust w.r.t. large variations in longitudinal speed, payload and road adhesion and w.r.t. unstructured uncertainty (unmodeled dynamics) as well. Moreover, its actions should not be uncomfortable for the driver and passengers. A steering controller structure that effectively satisfies the requirements outlined above is presented in this paper along with its associated design and analysis procedures in the context of automobile yaw dynamics improvement. The organization of the rest of the paper is as follows. The linearized single track vehicle yaw dynamics model is introduced in the appendix together with a model of an electric motor used as steering actuator. In section 2 a 
more detailed specification of the yaw dynamics improvement problem is given. The disturbance observer based steering controller is introduced in section 3 where design and robust stability requirements for unstructured uncertainty are also given as initial design guidelines. Weighted sensitivity, complementary sensitivity frequency domain constraints and eigenvalue specifications are mapped into controller parameter space in section 4 for final controller design. Simulation results based on the linear model in the appendix are used to demonstrate the effectiveness of the method in section 5 . In addition, considering the possibility of steering rate limitation, a realistic actuator model is used in a Popov criterion based nonlinear analysis in parameter space to investigate its effect on stability in section 6 .

\section{PROBLEM SPECIFICATIONS}

For modeling the vehicle yaw dynamics, the common linearized single track model is employed as a parametric linear time invariant plant (parametric LTI). The states are the yaw rate $r$ and the chassis side slip angle $\beta$. See A. 1 in the appendix for details of the model. Also model data corresponding to a mid size passenger car is given there. The variable exhibiting the largest variation during operation is the longitudinal speed $v$ (assumed to be varying slowly). The tire cornering stiffnesses $c_{f}$ and $c_{r}$ can also exhibit large variations due to variations in friction coefficient $\mu$ between the road and the tires. In addition to the single track model dynamics, there is a steer-by-wire actuator that is used to set the front wheel steering angle. The steering actuator model assumed is described in section A.2.

The longitudinal speed $v$ is assumed to vary between zero and a maximum value of $50 \mathrm{~m} / \mathrm{s}$ during operation. Gain scheduling will be necessary to softly switch on the controller with increasing speed starting at $10 \mathrm{~m} / \mathrm{s}$. However, in this paper only high speed operation is investigated at two exemplary speeds: $v=30 \mathrm{~m} / \mathrm{s}$ and $v=50 \mathrm{~m} / \mathrm{s}$. The assumed operating domain of the vehicle in terms of the speed $v$ and the road adhesion coefficient $\mu$ is displayed in Fig. 1. The maximum value of $\mu$ is one (dry road) at all speeds while its assumed minimum value increases linearly from 0.2 (icy road) at low speeds up to 0.8 (wet road) at high speeds. (Additional uncertainties will be considered in sections 3.1 and 4.2.) Cross markers in Fig. 1 indicate specific operating points which will be particularly looked at later.

The aim in yaw dynamics controller design is to make sure that stable and improved yaw dynamics is achieved for all operating conditions and all possible values of the uncertain parameters

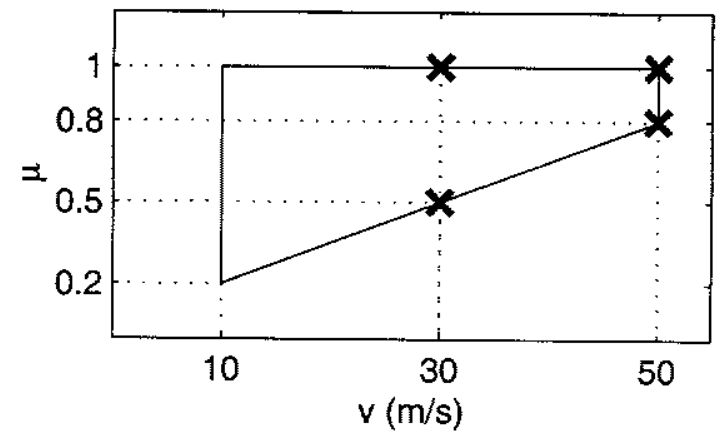

Fig. 1. Operating domain and considered operating points

despite the presence of additional unstructured uncertainty. Here, improved yaw dynamics means good disturbance rejection properties where the possible disturbances include the effect of wind forces and $\mu$-split braking. Good steering tracking performance is required as well. A disturbance observer based steering controller is designed and shown to effectively achieve the desired aim in the following sections.

\section{THE DISTURBANCE OBSERVER}

The disturbance observer is a specific method of designing a two degree of freedom control architecture to achieve insensitivity to both modeling errors and disturbance rejection (Ohnishi, 1987; Umeno and Hori, 1991). It has been used successfully in a variety of motion control applications including high speed direct drive positioning (Kempf and Kobayashi, 1999) and friction compensation (Güvenç and Srinivasan, 1994). The disturbance observer can also be used to achieve dynamics similar to those obtained by the use of inner feedback loops through control (Güvenç, 1999). The application of the disturbance observer control scheme to vehicle yaw dynamics control is introduced in the following.

The vehicle model can also be expressed as

$$
r=G \delta_{f}+d=\left(G_{n}\left(1+\Delta_{m}\right)\right) \delta_{f}+d
$$

where $G$ is the actual vehicle dynamics inputoutput relation between steering wheel angle $\delta_{f}$ and yaw rate $r . \Delta_{m}$ is the multiplicative model uncertainty in our knowledge of an adopted nominal model $G_{n}$ and $d$ is the external disturbance. The aim in disturbance observer design is to approximately obtain

$$
\frac{r}{\delta_{s}}=G_{n} G_{a}
$$

(compare to (A.7)) as the input-output relation (steering transfer function) despite the presence of model uncertainty $\Delta_{m}$ and external disturbance $d$ ( $\delta_{s}$ is the new input i.e. the steering wheel angle). 
This aim is achieved by treating the external disturbance and model uncertainty as an extended disturbance $e$ in disturbance observer design and solving for it as

$$
\begin{aligned}
& r=G_{n} \delta_{f}+\left(G_{n} \Delta_{m} \delta_{f}+d\right)=G_{n} \delta_{f}+e \\
& e=r-G_{n} \delta_{f}
\end{aligned}
$$

and using the new control signal $\delta_{s}$ given by

$$
\delta_{f}=G_{a}\left(\delta_{s}-\frac{e}{G_{n}}\right)=G_{a}\left(\delta_{s}-\frac{1}{G_{n}} r+\delta_{f}\right)
$$

to approximately cancel its effect in (3). Substituting (5) into (3) shows that the desired steering transfer function (2) is achieved if a good actuator $\left(G_{a} \rightarrow 1\right)$ is used. The front wheel steering angle $\delta_{f}$ is assumed to be the output of the steering actuator $G_{a}$. With the aim of trying to limit the compensation to a preselected low frequency range (in an effort not to overcompensate at high frequencies), the feedback signals in (5) are multiplied by the low pass filter $Q$ to obtain the implementation equation

$$
\delta_{f}=G_{a}\left(\delta_{s}-\frac{Q}{G_{n}} r+Q \delta_{f}\right)
$$

which can also be seen in the block diagram of Fig. 2. Including $G_{a}$ in the inner feedback loop

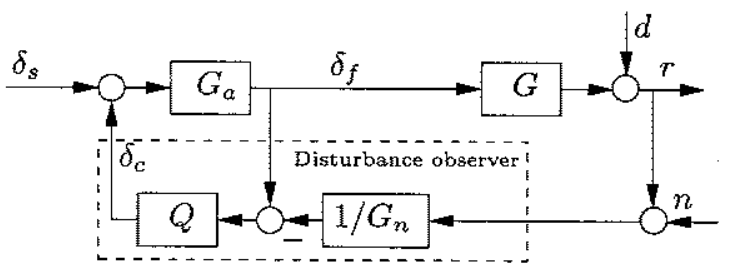

Fig. 2. Closed loop structure with disturbance observer.

helps in reducing the effect of actuator saturation on disturbance observer performance (Aksun Güvenç and Güvenç, 2002). The relative degree of the unity d.c. gain low pass filter $Q$ is chosen to be at least equal to the relative degree of $G_{n}$ for causality of $Q / G_{n}$. In the sequel, the structure of $Q$ is assumed to be

$$
Q=\frac{1}{\tau_{Q} s+1}
$$

The nominal steering transfer function is chosen as a first order system here given by

$$
G_{n}(s)=\frac{K_{n}(v)}{\tau_{n} s+1}
$$

and $K_{n}(v)$ is the steady state gain of the nominal single track model (i.e. on dry road, $\mu_{n}=1$ ) at the actual longitudinal speed $v$ (see (A.6) in the appendix).

The open loop transfer function at signal $r$ of the disturbance observer compensated yaw dynamics model is

$$
L:=\frac{G G_{a} Q}{G_{n}\left(1-G_{a} Q\right)}
$$

The steering transfer function, disturbance rejection (i.e. sensitivity function $S$ ) and sensor noise rejection (i.e. complementary sensitivity function T) transfer functions given are then

$$
\begin{aligned}
\frac{r}{\delta_{s}}=\frac{G_{n} G_{a} G}{G_{n}\left(1-G_{a} Q\right)+G_{a} G Q} \\
S:=\frac{r}{d}=\frac{1}{1+L}=\frac{G_{n}\left(1-G_{a} Q\right)}{G_{n}\left(1-G_{a} Q\right)+G_{a} G Q} \\
T:=-\frac{r}{n}=\frac{L}{1+L}=\frac{G_{a} G Q}{G_{n}\left(1-G_{a} Q\right)+G_{a} G Q}
\end{aligned}
$$

from which it is obvious that for good performance $Q$ must be a unity gain low pass filter $\left(G_{a}\right.$ is a unity gain low pass filter as well). This choice will result in $r / \delta_{s} \rightarrow G_{n}, r / d \rightarrow 0$ at low frequencies where $Q \rightarrow 1$ and $r / n \rightarrow 0$ at high frequencies where $Q \rightarrow 0$ as is desired. Disturbance observer design is thus mainly shaping the filter $Q$ to satisfy the design objectives. The first limitation on the bandwidth of $Q$ comes from the sensor noise rejection at sensor noise frequencies. The second limitation is that the bandwidth of $Q$ should not be larger than the bandwidth of the actuator used as it makes no sense to command what cannot be achieved.

\subsection{Stability robustness}

Another bandwidth limitation for the $Q$ filter comes from the robust stability criterion

$$
|Q|<\left|\frac{1}{\Delta}\right| \equiv\left|\frac{1}{G_{a} \Delta_{m}}\right|, \forall \omega
$$

in the presence of unstructured multiplicative model uncertainty $\Delta_{m}$. The disturbance observer design requirements specified in terms of the filter $Q$ are summarized in Fig. 3. For the uncertainty

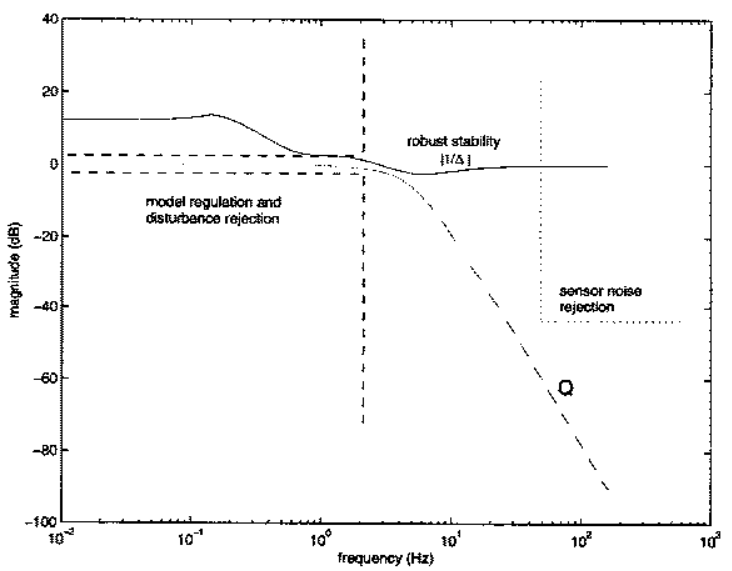

Fig. 3. Disturbance observer design specifications in the mass, moment of inertia and front and rear 
cornering stiffness considered, the corresponding $|1 / \Delta|$ profiles were calculated at several different values of the longitudinal speed $v \in[30,50] \mathrm{m} / \mathrm{s}$. For the nominal steering transfer function $\tau_{n}=$ $0.165 \mathrm{~s}$ was used. The mass $m \in[1296,1696] \mathrm{kg}$ and moment of inertia $J \in[1750,2100] \mathrm{kg} \mathrm{m}^{2}$ were assumed to be linearly related according to $J=616 \mathrm{~kg} \mathrm{~m}^{2}+\left(0.875 \mathrm{~m}^{2}\right) m$ in the calculations. The composite lower bound obtained from this calculation is displayed as the upper robust stability boundary in Fig. 3 from which can be seen that $Q$ should have a maximum bandwidth of 8 $\mathrm{Hz}$.

The tuning of $\tau_{Q}$ and $\tau_{n}$ in the controller (eqs. $(6),(7),(8))$ in order to achieve robust matching of the design objectives is the scope of the following section.

\section{PARAMETER SPACE DESIGN}

In this section, the parameter space approach is applied for robust controller design and analysis. Besides eigenvalue specifications ( $\Gamma$-stability) also Bode magnitude type specifications ( $\mathcal{B}$ - stability) on sensitivity functions are incorporated into the controller design to enhance vehicle stability as well as performance and handling qualities robustly w.r.t. structured $(v, \mu)$ and unstructured uncertainty ( $m, J$, unmodeled dynamics).

The section is subdivided into four subsections. In section 4.1 the problem setup is stated. Section 4.2 describes the stability and performance specifications in eigenvalue and frequency domain for controller design. In section 4.3 , the parameter space approach is used to map these specifications into the plane of the two controller parameters $\tau_{Q}$ and $\tau_{n}$ and in section 4.5 the resulting controller is analyzed in terms of sensitivity.

\subsection{Problem setup}

Consider Fig. 2 and (7). $Q$ is chosen as a unity d.c. gain first order low pass filter with time constant $\tau_{Q}$. The open-loop single-input-singleoutput transfer function $L$, sensitivity function $S$ and complementary sensitivity function $T$ are given by (9), (11) and (12), respectively. Furthermore, the characteristic polynomial of the closed loop system is defined as

$$
p:=\text { Numerator }\{1+L\} .
$$

In the following demonstration of the design procedure applying $\Gamma$ - and $\mathcal{B}$-stability, the time constants $\tau_{n}$ of the nominal model $G_{n}$ in (8) and $\tau_{Q}$ of the filter $Q$ in (7), respectively, are considered as controller parameters. These time constants shall be tuned such that the feedback provides $\Gamma$ - and
$\mathcal{B}$-stability for the whole operating domain given in Fig. 1. In a first design step being described in this paper, only the four operating conditions marked by crosses in Fig. 1 are considered. For each of them the $\Gamma$ - and $\mathcal{B}$-stability specifications given below are mapped into the controller parameter plane $\left(\tau_{n}, \tau_{Q}\right)$.

\subsection{Control design specifications}

\section{Г-stability}

Hurwitz-stability requires that all roots of the characteristic polynomial $p(s)$ lie in the complex left half plane $\mathbb{C}^{-}$, i.e.

$$
\operatorname{roots}\{p(s)\} \subset \mathbb{C}^{-} .
$$

A more generalized stability notion is $\Gamma$-stability (Ackermann et al., 1993; Ackermann et al., 2002) where also other eigenvalue specifications w.r.t. the eigenvalue location can be formulated. A system is called $\Gamma$-stable if all roots of $p$ are located within a predefined region $\Gamma$ being bounded by $\partial \Gamma$, i.e.

$$
\operatorname{roots}\{p(s)\} \subset \Gamma \subseteq \mathbb{C} .
$$

Fig. 4 shows the $\Gamma$-region representing the eigenvalue specifications which are one part of multiple specifications for the design of $\tau_{n}$ and $\tau_{q}$ for all $(\mu, v)$ in the operating domain. The boundary $\partial \Gamma$

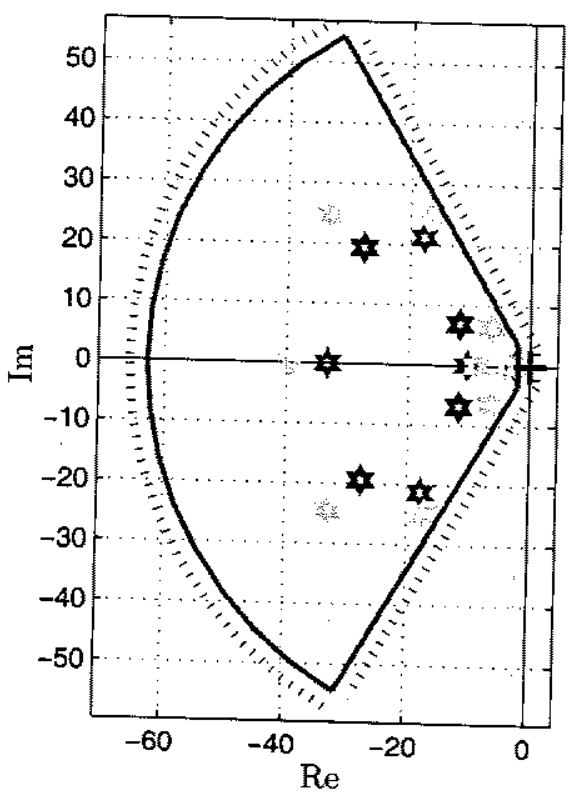

Fig. 4. $\Gamma$-specifications in eigenvalue domain.

of $\Gamma$ is composed of three geometric elements. The first boundary is a shifted imaginary axis $s=-2$ which ensures that the system's settling time is limited. Two lines of constant damping guarantee a minimum damping of 0.5 of all poles, and a circle centered at the origin with radius $R=2 \pi 10$ 
guarantees that the natural frequency of any pole does not exceed $10 \mathrm{~Hz}$. The poles of the closed loop system shall be located in the admissable eigenvalue region $\Gamma$ as shown in Fig. 4, i.e.

$$
\operatorname{roots}\left\{p\left(s, \tau_{n}, \tau_{Q}, v, \mu\right)\right\} \subset \Gamma .
$$

\section{$\mathcal{B}$-stability}

The notion of $\mathcal{B}$-stability (Odenthal and Blue, 2000; Ackermann et al., 2002) is analogous to that of $\Gamma$-stability. However, frequency response magnitude (FRM) specifications are used for $\mathcal{B}$ stability definition. A system is considered to be $\mathcal{B}$-stable if its FRM lies within a desired region in the FRM plot (i.e. the Bode magnitude plot) referred to as $\mathcal{B}$. $\mathcal{B}$-stability shall now be applied to bound the Bode magnitude plots of the sensitivity function $S$ given by (11) and the complementary sensitivity function $T$ given by (12), i.e. weighting functions $W_{S}(s)$ and $W_{T}(s)$ are selected such that

$$
\begin{array}{ll}
|S(j \omega)|<\left|W_{S}(j \omega)^{-1}\right|, & \forall \omega \\
|T(j \omega)|<\left|W_{T}(j \omega)^{-1}\right|, & \forall \omega .
\end{array}
$$

These inequalities are well known as the "nominal performance" and the "robust stability" criteria (assuming a multiplicative uncertainty model) (Doyle et al., 1992)

Disturbance rejection a) To ensure disturbance attenuation at low frequencies $|S(j \omega)| \ll 1$ must hold. b) However, since $L$ is a robustly stable transfer function with a relative degree of three, Bode's integral theorem applies which means that attenuating disturbances at low frequencies unavoidably results in amplifying them at high frequencies. Therefore, also the amplification must be restricted to an acceptable level, i.e. $\|S(j \omega)\|_{\infty}<1 / \rho_{s}$, where $\rho_{S}$ is equivalent to the required Nyquist stability margin.

These two specifications a) and b) are combined into one specification by selecting an upper bound on the FRM of $S$ (see Fig. 9), i.e.

$$
\left|S\left(j \omega, \tau_{n}, \tau_{Q}, v, \mu\right)\right|<\left|W_{S}(j \omega)^{-1}\right|, \quad \forall \omega
$$

where

$$
W_{s}(s)^{-1}=1.8 \frac{s+0.7}{s+12.6} \text {. }
$$

Robustness w.r.t. unstructured uncertainty Two magnitude bounds on $T$, i.e. the magnitudes of $W_{T, 1}(s)^{-1}$ and $W_{T, 2}(s)^{-1}$, are selected to capture robustness w.r.t. unstructured uncertainty, i.e. using a multiplicative uncertainty model, subject to unmodeled dynamics and uncertainty in mass $m$ and moment of inertia $J$. a) The disturbance observer shall guarantee robustness to $10 \%$ magnitude uncertainty at low frequencies, i.e. where the model of the vehicle and the actuator is reasonably accurate, and $500 \%$ uncertainty at high frequencies, i.e. where unmodeled dynamics come into play. Thereby, a transition frequency of $6 \mathrm{~Hz}$ between low gain and high gain of the weighting function $W_{T_{1}}(s)$ was selected based on the knowledge of the vehicle model's accuracy and the performance specification of the steering actuator.

b) A second bound on $T$, i.e. $W_{T, 2}(s)^{-1}$, is used to cover the disturbance observer stability specifcations subject to model uncertainty in $m$ and $J$ as outlined in section 3 .

Hence, the following $\mathcal{B}$-specifications will be used as constraints for $T$ :

$\left|T\left(j \omega, \tau_{n}, \tau_{Q}, v, \mu\right)\right|<\left|W_{T, i}(j \omega)^{-1}\right|, \forall \omega, i=1,2$,

where

$$
\begin{aligned}
& W_{T, 1}(s)=5 \frac{s+3.77}{s+188.5} \\
& W_{T, 2}(s)=\frac{0.12804(s+43.98)(s+0.4833)}{(s+6.124)(s+2.882)}
\end{aligned}
$$

(see Fig. 10). Note, that (20) represents the "robust stability" criterion for two different multiplicative uncertainty models. At low frequencies unstructured uncertainty subject to uncertainty in $m$ and $J$ is relevant and for high frequencies uncertainty subject to unmodeled dynamics.

\subsection{Mapping of $\Gamma$-and $\mathcal{B}$-stability boundaries into parameter space}

The mapping of the $\Gamma$-stability boundaries defined in section 4.2 is based on the limit condition that the characteristic polynomial $p\left(s, \tau_{n}, \tau_{q}, v, \mu\right)$ has a root exactly on the $\Gamma$-region boundary $\partial \Gamma$. Therefore, the condition $\partial \Gamma=s(\alpha)=\sigma(\alpha)+$ $j \omega(\alpha)$, where $\alpha$ is the parameterization of $\partial \Gamma$, is substituted into the characteristic polynomial given by (14) which is seperated into two equations for its real and imaginary part respectively:

$$
\begin{aligned}
& \operatorname{Re} p\left(\alpha, \tau_{n}, \tau_{Q}, v, \mu\right)=0 \\
& \operatorname{Im} p\left(\alpha, \tau_{n}, \tau_{Q}, v, \mu\right)=0
\end{aligned}
$$

The mapping of the $\Gamma$-stability boundaries into the parameter plane $\left(\tau_{n}, \tau_{Q}\right)$ requires algebraic solution of these two equations (Ackermann et al., 1993; Ackermann et al., 2002; Kaesbauer, 2000). Fig. 5 shows the result for the mapping of the $\Gamma$-stability boundaries (as defined in Fig. 4) for the two operating conditions with $v=50 \mathrm{~m} / \mathrm{s}$ and $\mu \in\{0.8,1\}$ (see Fig. 1). The $\left(\tau_{n}, \tau_{Q}\right)$ region which simultaneously $\Gamma$-stabilizes the two operating points is denoted $K_{\Gamma_{1}}$. In this plot and 
also in Fig. 6 and Fig. 7 the gray lines correspond to the operating condition with $\mu=0.8$, and the black lines to that with $\mu=1$. For a better distinction between $\Gamma$ - and $\mathcal{B}$-stability boundaries, dashed linestyle is used for $\Gamma$-stability in this plot and in the subsequent plots. For the sake of consciseness the $\Gamma$-stability boundaries for $v=$ $30 \mathrm{~m} / \mathrm{s}$ and $\mu \in\{0.5,1\}$ are not displayed in Fig. 5, Fig. 6 and Fig. 7.

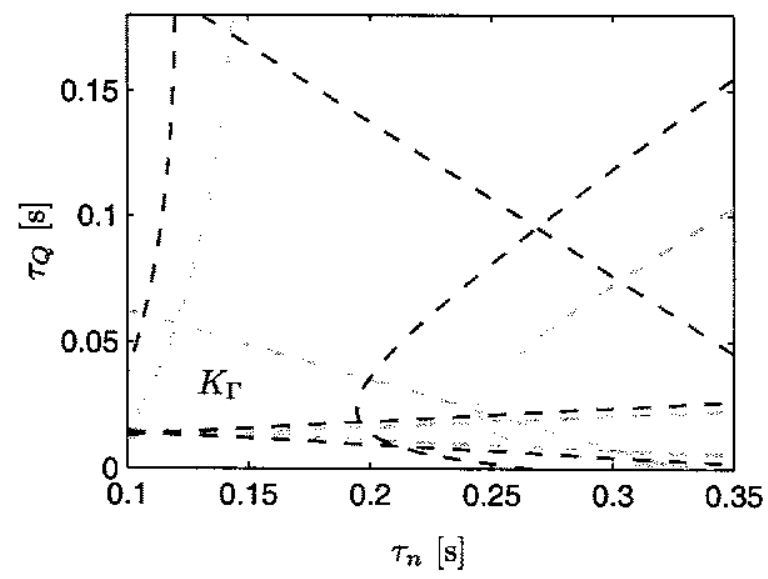

Fig. 5. $\Gamma$-stable region for $v=50 \mathrm{~m} / \mathrm{s}$ and $\mu \in$ $\{0.8,1\}$.

To establish the mapping equations of the $\mathcal{B}$ stability boundaries defined by eqs. (18) and (20), it suffices to consider two mathematical conditions, the point and the tangent condition (Odenthal and Blue, 2000). The point condition applies when $\left|S\left(j \omega, \tau_{n}, \tau_{Q}, v, \mu\right)\right| \quad$ or $\left|T\left(j \omega, \tau_{n}, \tau_{Q}, v, \mu\right)\right|$ respectively starts $(\omega=0)$ or ends $(\omega \rightarrow \infty)$ on the boundary $\partial \mathcal{B}_{S}=$ $\left|W_{S}(j \omega)^{-1}\right|$ or $\partial \mathcal{B}_{T, i}=\left|W_{T, i}(j \omega)^{-1}\right|$ respectively. The tangent condition allows for the mapping of touching points, i.e. the points where $\left|S\left(j \omega, \tau_{n}, \tau_{Q}, v, \mu\right)\right|$ or $\left|T\left(j \omega, \tau_{n}, \tau_{Q}, v, \mu\right)\right|$ respectively becomes tangent to $\partial \mathcal{B}_{S}$ or $\partial \mathcal{B}_{T, i}$ respectively. Details about the mapping of $\mathcal{B}$ specifications are described in (Odenthal and Blue, 2000; Ackermann et al., 2002).

To ensure that the magnitude of the sensitivity function $\left|S\left(j \omega, \tau_{n}, \tau_{Q}, v, \mu\right)\right|$ remains below its upper bound, condition (18) is mapped to the $\left(\tau_{n}, \tau_{k}\right)$ controller parameter plane. Fig. 6 shows the resulting region $K_{\mathcal{B}_{S}}$ for the two operating points at $v=50 \mathrm{~m} / \mathrm{s}$ with $\mu \in\{0.8,1\}$. For any parameter combination $\left(\tau_{n}, \tau_{Q}\right)$ taken from this region condition (18) is satisfied for both operating points simultaneously.

For consideration of robust stability, condition (20) is mapped to the $\left(\tau_{n}, \tau_{Q}\right)$ controller parameter plane (see Fig. 7). The dotted lines correspond to the Hurwitz-stability condition, i.e. the nominal stability condition, for the two operating points considered. This nonconservative mapping

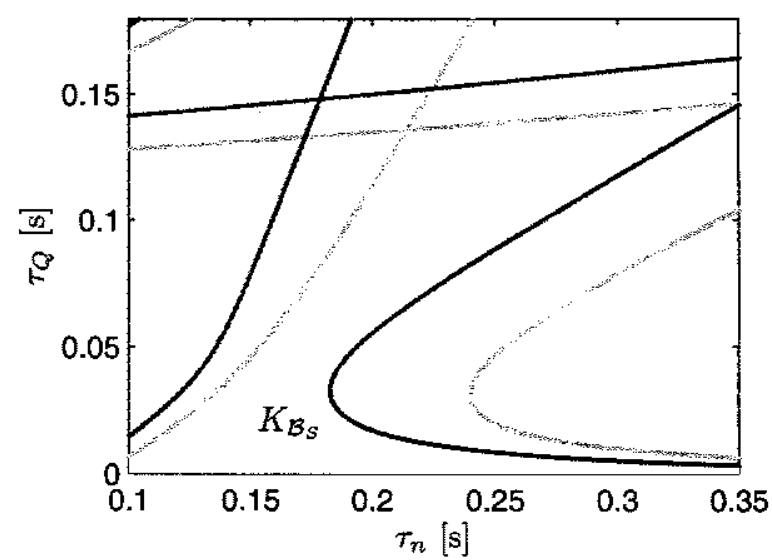

Fig. 6. Nominal performance in parameter space for $v=50 \mathrm{~m} / \mathrm{s}$ and $\mu \in\{0.8,1\}$.

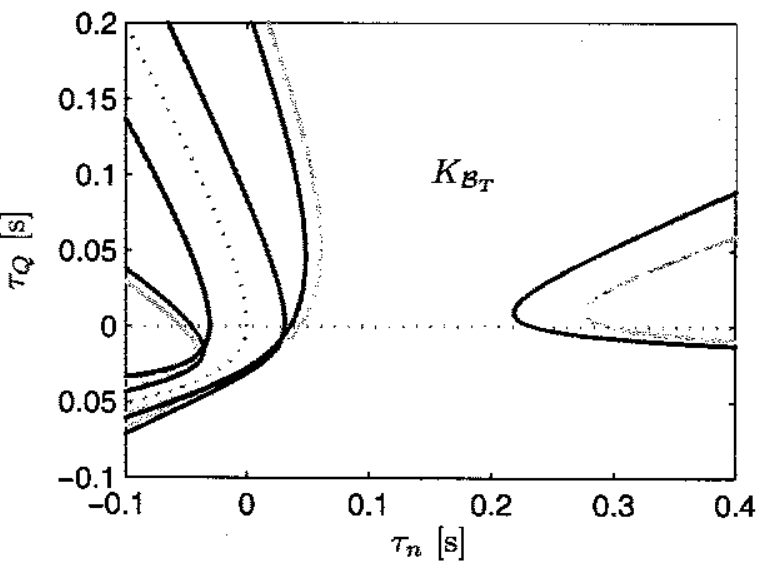

Fig. 7. Robust stability in parameter space for $v=50 \mathrm{~m} / \mathrm{s}$ and $\mu \in\{0.8,1\}$.

shows which set of $\left(\tau_{n}, \tau_{Q}\right)$ gains (i.e. the region $K_{\mathcal{B}_{T}}$ in Fig. 7) will guarantee robust stability in the presence of actuator uncertainty, unmodeled dynamics and/or structured uncertainty due to the disturbance observer specifications simultaneously for the two operating points at $v=50 \mathrm{~m} / \mathrm{s}$ with $\mu \in\{0.8,1\}$. Similiar results were achieved for the other two operating points $v=30 \mathrm{~m} / \mathrm{s}$ and $\mu \in\{0.5,1\}$.

The final step in controller design is superimposing the previously determined stability regions in parameter space. The intersection of all of the individual regions in the $\left(\tau_{n}, \tau_{Q}\right)$ plane, which satisfy the formulated $\Gamma$ - and $\mathcal{B}$-specifications, for all four operating points form the desired parameter region $K$ shown in Fig. 8. For any choice of $\left(\tau_{n}, \tau_{\dot{Q}}\right) \in K$, all previously described requirements in terms of $\Gamma$ - and $\mathcal{B}$-stability are met simultaneously for the four operating conditions. 


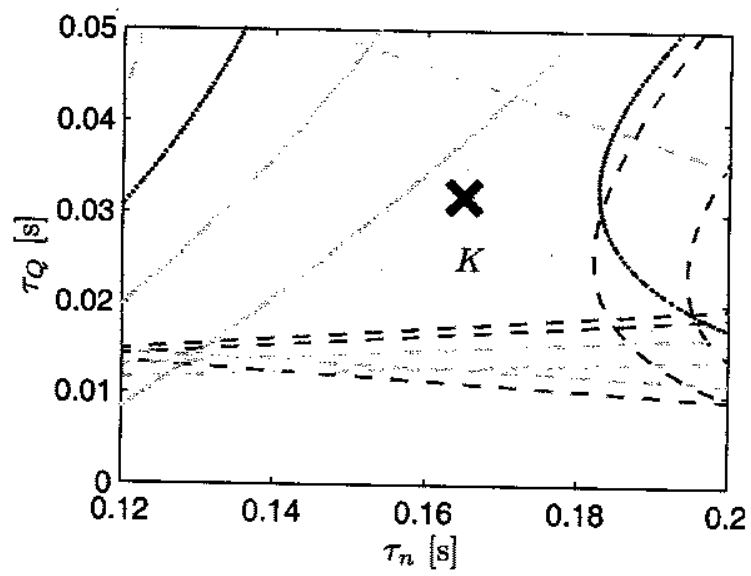

Fig. 8. Parameter region which meets all $\mathcal{B}$ - and $\Gamma$-specifications for $v=50 \mathrm{~m} / \mathrm{s}, \mu \in\{0.8,1\}$ and $v=30 \mathrm{~m} / \mathrm{s}, \mu \in\{0.5,1\}$.

\subsection{Controller selection}

Note that satisfying all specifications postulated in section 4.2 also for low speed (e.g. $v=10$ $\mathrm{m} / \mathrm{s}$ ) requires gain scheduling of $\tau_{n}$ and $\tau_{Q}$ with speed. This is not considered here. Instead, from the region $K$ in Fig. 8 the parameters $\tau_{n}=0.165 \mathrm{~s}$ and $\tau_{Q}=0.0318 \mathrm{~s}$ are chosen as fixed controller parameters (marked with a cross). Fig. 4 shows all eigenvalues of the closed loop system for the four operating conditions. They are all included in the desired $\Gamma$-region.

\subsection{Sensitivity analysis}

Figs. 9 and 10 show $|S(j \omega)|$ and $|T(j \omega)|$ for the four operating points. They all remain below $\left|W_{S}(j \omega)^{-1}\right|,\left|W_{T, 1}(j \omega)^{-1}\right|$ and $\left|W_{T, 2}(j \omega)^{-1}\right|$ respectively, i.e. they are entirely included in the $\mathcal{B}$-stable regions.

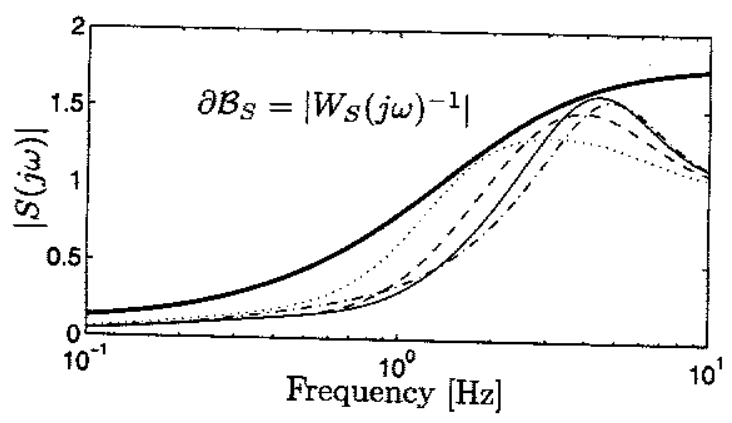

Fig. 9. Frequency response magnitude plot of the sensitivity function.

\section{SMMULATION RESULTS}

Two versions of the vehicle are compared in this section by means of linear simulation: The conventional car and the controlled car. For the sake of

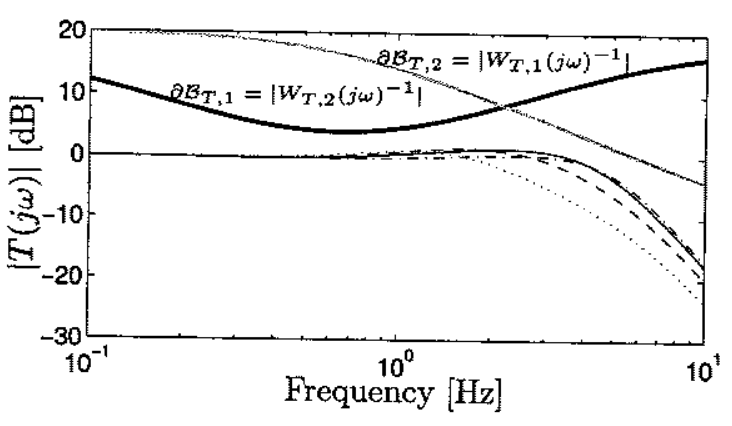

Fig. 10. Frequency response magnitude plot of the complementary sensitivity function.

comparability, the conventional car is assumed to be a steer-by-wire vehicle equipped with the same steering actuator as the controlled car (see (A.7)). The steering transfer function of the controlled car is given by (10). The controller parameters are set according to the design results in section $4: \tau_{n}=$ $0.165 \mathrm{~s}$ and $\tau_{Q}=0.0318 \mathrm{~s}$. Two maneuvers are investigated: A steering wheel step input and a yaw disturbance torque step input. The maneuvers are performed at the four operating points marked in Fig. 1. The results are shown in Figs. 11-14 in terms of the yaw rate $r$, the steering wheel angle $\delta_{s}$ and the front wheel steering angle $\delta_{f}$.
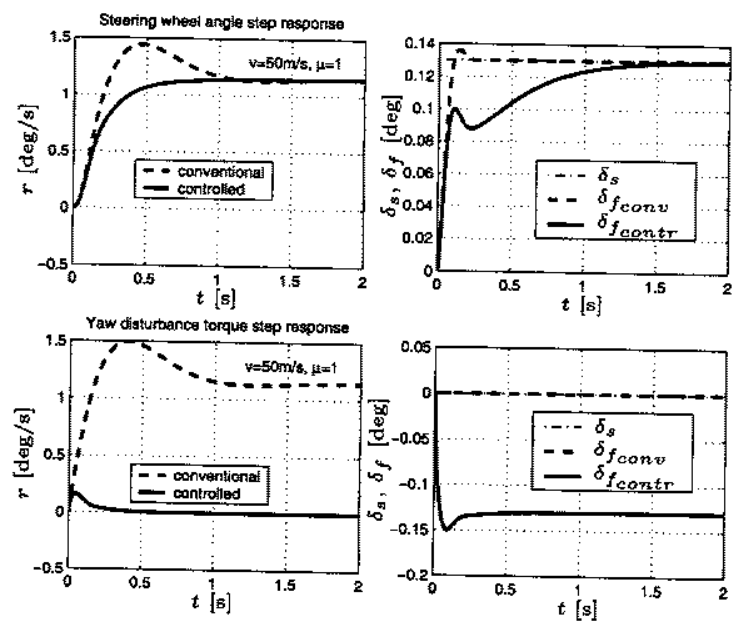

Fig. 11. Linear simulation results at $v=50 \mathrm{~m} / \mathrm{s}$, $\mu=1$.

The simulations show that the controller provides excellent disturbance rejection and a good steering tracking at all investigated operating points. The yaw disturbances are robustly attenuated within half a second. The control action is finished before the driver is even capable of starting his countersteering due to his reaction time of about a second. The yaw rate responses to a steering wheel input are well in accordance with the desired steering transfer function (2) not exhibiting any overshoot. Zero steady state error is inherent to the control structure as already discussed by means of (10)-(12). The steady state gain of the controlled vehicle steering transfer function is $K_{n}(v)$. This 

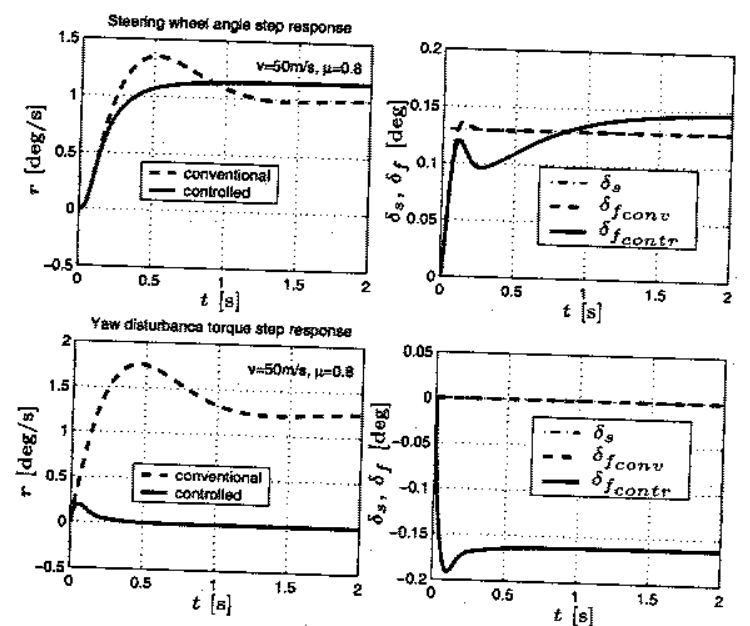

Fig. 12. Linear simulation results at $v=50 \mathrm{~m} / \mathrm{s}$, $\mu=0.8$.
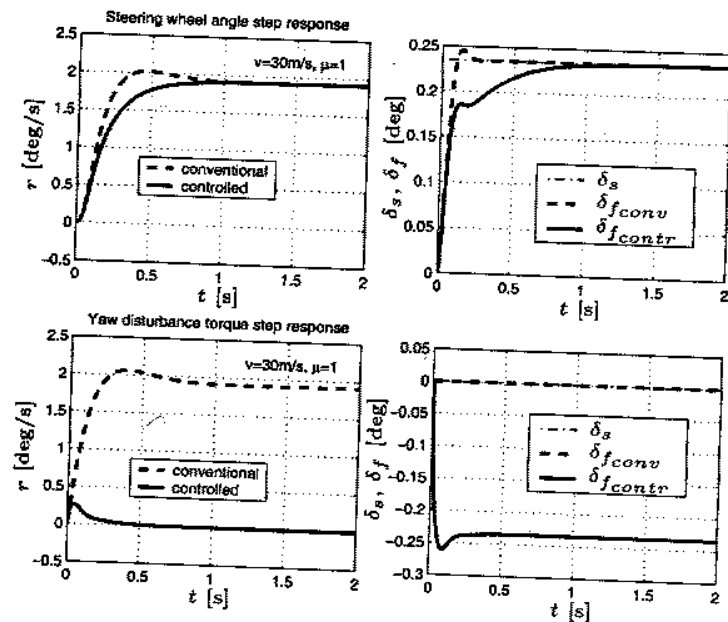

Fig. 13. Linear simulation results at $v=30 \mathrm{~m} / \mathrm{s}$, $\mu=1$.
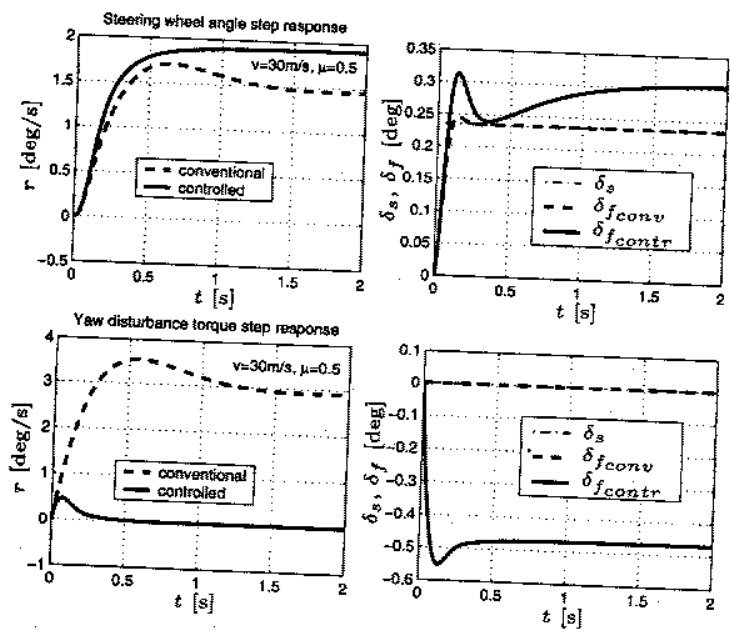

Fig. 14. Linear simulation results at $v=50 \mathrm{~m} / \mathrm{s}$, $\mu=0.5$. gain has been calculated to be identical to the conventional vehicle steady state gain for $\mu_{n}=1$. Therefore, the steady state yaw rate of the conventional vehicle and the controlled vehicle after a steering wheel input are identical for $\mu=1$ (Fig. 11 and Fig. 13) but differ otherwise.

Note that other choices for $Q$ and $G_{n}$ than given by (7) and ( 8 ) are feasible and may be reasonable depending on the specifications. For example a second order filter for $G_{n}$ to represent a certain desired single track steering transfer function can be used. If this model is well in accordance with the actual vehicle, then the steering transfer function is not significantly changed by control. However, good disturbance rejection is retained. On the other hand, a band-pass filter can be applied for $Q$ instead of a low pass filter. This removes control action at low frequencies ("fading controller") and transfers the task of compensating steady state errors back to the driver (Aksun Güvenc et al., 2001).

\section{NONLINEAR STABILITY ANALYSIS}

Previous experimental experience shows that limit cycles with actively steered cars can occur due to actuator nonlinearities (Ackermann and Bünte, 1999). In this paper, the rate limitation of the steering actuator is considered in terms of the limitation of the electric voltage of the motor (see Appendix A.2 for details of the steering actuator model). The Popov criterion (Popov, 1962) is employed to prove absolute stability for the controlled car such that limit cycles can not occur in the whole operating domain of the car. The foundations of mapping the Popov criterion to parameter space are described in (Bünte, 2000; Ackermann et al., 2002). Any criteria referring to frequency loci are subsumed with the notion of $\Theta$-stability. With the Popov absolute stability criterion, the Popov plot of the system's linear part is considered, whereas for the static timeinvariant nonlinear part only a sector needs to be known which includes the nonlinearity characteristics. The transfer function of the system's linear part is the transfer function from signal $u$ to signal $-u_{1}$ in Fig. A.2. Therefore, the actuator is considered to be connected to the control loop as shown in Fig. 2 with $\delta_{f r e f}=\delta_{s}+\delta_{c}$. The Popov plot is derived from the locus of the linear part transfer function, whereupon its imaginary part is multiplied with the respective frequency. The saturation in Fig. A.2 is a characteristic which is included in a sector formed by the two straight lines $u=0 \cdot u_{1}$ and $u=k \cdot u_{1}$ with $k=1$. According to the Popov criterion absolute stability is proven if the linear part is Hurwitz and the Popov plot lies to the right hand side of the socalled Popov 


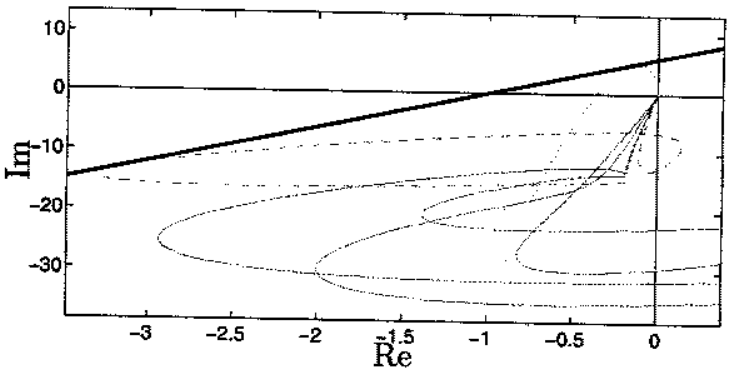

Fig. 15. Popov line (black) and Popov plots (gray) for six operating conditions marked in Fig. 16.

line. (This definition-is adopted for $\Theta$-stability here.) The Popov line intersects the real axis at $-1 / k=-1$. The slope $M$ of the Popov line may be chosen arbitrarily. We chose $M=6.0$ (see Fig. 15) which turns out to be favorable in terms of the resulting $\Theta$-stable parameter region $Q_{\ominus}$. Thus, the whole operating domain of the vehicle is included in $Q_{\ominus}$ as illustrated in Fig. 16. Fig. 15

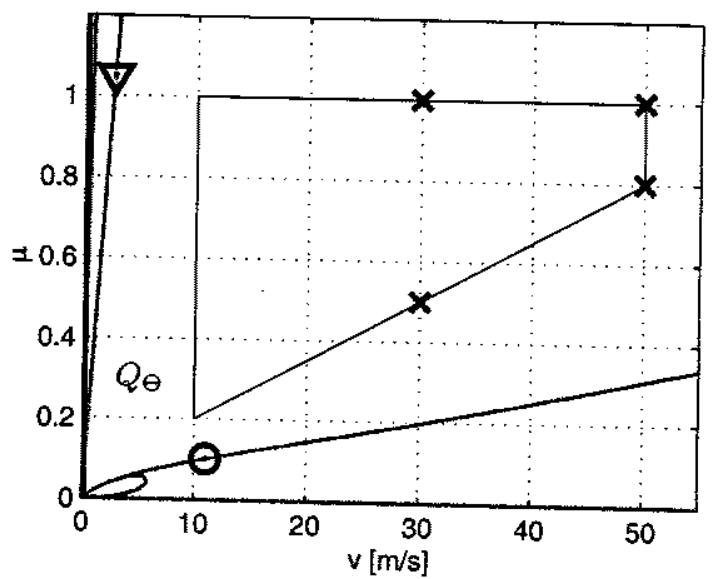

Fig. 16. Region $Q_{\Theta}$ of absolute stability using a Popov line with slope $M=6.0$.

also shows six Popov plots corresponding to six operating points marked in Fig. 16. Solid lines are used for the Popov plots of the operating points indicated by cross markers. The Popov plot belonging to the circular mark is plotted dashed, the one belonging to the triangular marker is plotted with dotted linestyle. The latter two cases are $\Theta$ limit-stable since the operating points are located on a $\Theta$-stability boundary in the $(v, \mu)$-plane. This illustrates the limit case of a Popov plot touching the Popov line which was used for mapping stability boundaries into parameter space. By this approach, the robust nonexistence of limit cycles in the operating domain is proven while accounting for the presence of the saturation nonlinearity. A similar proof, however applying the dual locus method, has been shown in (Ackermann and Bünte, 1999) for a different control structure.

\section{CONCLUSIONS}

A two degree of freedom steering controller based on the disturbance observer for vehicle yaw dynamics improvement was introduced here. For robust controller design, weighted sensitivity and complementary sensitivity frequency domain specifications ( $\mathcal{B}$-stability) and eigenvalue specifications ( $\Gamma$-stability) were mapped into the parameter space of controller parameters. Simulation results based on the linear single track model were used to demonstrate the achievement of excellent disturbance rejection and good steering command response. In addition, considering the possibility of steering actuator rate limitation due to electric voltage limitation, a Popov criterion based nonlinear analysis was performed for proving robust absolute stability. Therefore, respective $\Theta$-stability boundaries were mapped into a plane of operating parameters.

\section{REFERENCES}

Ackermann, J., A. Bartlett, D. Kaesbauer, W. Sienel and R. Steinhauser (1993). Robust control: Systems with uncertain physical parameters. Springer. London.

Ackermann, J. and T. Bünte (1999). Robust prevention of limit cycles for robustly decoupled car steering dynamics. Kybernetika 35(1), 105-116.

Ackermann, J., P. Blue, T. Bünte, L. Güvenc, D. Kaesbauer, M. Kordt, M. Muhler and D. Odenthal (2002). Robust Control: The Structural Approach. Springer. London. (to appear).

Aksun Güvenc, B., T. Bünte, D. Odenthal and L. Güvenc (2001). Robust two degree of freedom vehicle steering controller design. In: Proc. American Control Conference. Arlington, VA, USA.

Aksun Güvenç, B. and L. Güvenç (2002). Robust two degree-of-freedom add-on controller design for automatic steering. IEEE Trans, on Control Systems Technology 10(1), 137-148.

Bünte, T. (2000). Mapping of Nyquist/Popov Theta-stability margins into parameter space. In: Proc. 3rd IFAC Symposium on Robust Control Design. Prague, Czech Republic.

Doyle, J. C., B. A. Francis and A. R. Tannenbaum (1992). Feedback control theory. Macmillan Publishing Company. New York.

Güvenç, L. (1999). Closed loop pneumatic position control using discrete time model regulation. In: Proc. American Control Conference. pp. 4273-4277.

Güvenç, L. and K. Srinivasan (1994). Friction compensation and evaluation for a force control application. J. of Mechanical Systems and Signal Processing 8(6), 623-638. 
Kaesbauer, D. (2000), Solving nonlinear parametric mapping equations. In: Proc. 3rd IFAC Symposium on Robust Control Design. Prague, Czech Republic.

Kempf, C.J. and S. Kobayashi (1999). Disturbance observer and feedforward design for a high-speed direct-drive positioning table. IEEE Trans, on Control Systems Technology $7(5), 513-526$.

Odenthal, D. and P. Blue (2000). Mapping of frequency response magnitude specifications into parameter space. In: Proc. 3rd IFAC Symposium on Robust Control Design. Prague, Czech Republic.

Ohnishi, K. (1987). A new servo method in mechatronics. Trans. Japanese Soc. Electr. Eng. 107-D, 83-86.

Popov, V.M. (1962). Absolute stability of nonlinear systems of automatic control. Autom. $\&$ Rem. Control 22, 857-875.

Riekert, P. and T.E. Schunck (1940). Zur Fahrmechanik des gummibereiften Kraftfahrzeugs. Ingenieur Archiv 11, 210-224.

Umeno, T. and Y. Hori (1991). Robust speed control of dc servomotors using modern two degrees-of-freedom controller design. IEEE Trans. Ind. Electron. 38(5), 363-368.

\section{Appendix A. VEHICLE MODEL AND STEERING ACTUATOR MODEL}

\section{A.1 Vehicle yaw dynamics model}

The car model which is used for the investigations in this paper is the classical linearized single track model (Riekert and Schunck, 1940) as illustrated in Fig. A.1. Its major variables and geometric

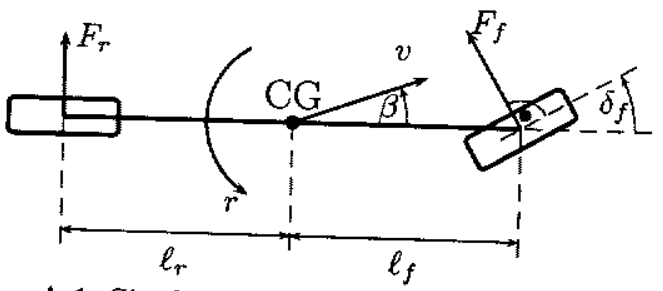

Fig. A.1. Single-track model

parameters are

\begin{tabular}{|c|c|}
\hline$F_{f}\left(F_{r}\right)$ & $\begin{array}{l}\text { lateral wheel force at } \\
\text { front (rear) wheel }\end{array}$ \\
\hline$r$ & yaw rate \\
\hline$\beta$ & $\begin{array}{l}\text { chassis side slip angle at } \\
\text { center of gravity (CG) }\end{array}$ \\
\hline$v$ & $\begin{array}{l}\text { magnitude of velocity vector } \\
\text { at } \mathrm{CG}(v>0, \dot{v}=0)\end{array}$ \\
\hline$\ell_{f}\left(\ell_{r}\right)$ & $\begin{array}{l}\text { distance from front (rear) } \\
\text { axle to } \mathrm{CG}\end{array}$ \\
\hline$\delta_{f}$ & front wheel steering angle \\
\hline
\end{tabular}

The mass of the vehicle is $m$ and $J$ is the moment of inertia w.r.t. a vertical axis through the CG. For small steering angle $\delta_{f}$ and small side slip angle $\beta$, the linearized equations of motion are (Ackermann et al., 1993; Ackermann et al., 2002)

$$
\left[\begin{array}{c}
m v(\dot{\beta}+r) \\
m \ell_{f} \ell_{r} \dot{r}
\end{array}\right]=\left[\begin{array}{c}
F_{f}+F_{r} \\
F_{f} \ell_{f}-F_{r} \ell_{r}
\end{array}\right]
$$

The tire force characteristics are linearized as

$$
F_{f}\left(\alpha_{f}\right)=\mu c_{f 0} \alpha_{f}, \quad F_{r}\left(\alpha_{r}\right)=\mu c_{r 0} \alpha_{r}
$$

with the tire cornering stiffnesses $c_{f 0}, c_{r 0}$, the road adhesion factor $\mu$, and the tire side slip angles

$$
\begin{aligned}
& \alpha_{f}=\delta_{f}-\left(\beta+\frac{\ell_{f}}{v} r\right) \\
& \alpha_{r}=-\left(\beta-\frac{\ell_{r}}{v} r\right)
\end{aligned}
$$

The transfer function from the front wheel steering angle $\delta_{f}$ to the yaw rate $r$ can be computed from (A.1)-(A.4):

$$
G(s)=\frac{r(s)}{\delta_{f}(s)}=\frac{b_{0}+b_{1} s}{a_{0}+a_{1} s+a_{2} s^{2}}
$$

with

$$
\begin{aligned}
b_{0} & =c_{f} c_{r}\left(l_{f}+l_{r}\right) v \\
b_{1} & =c_{f} l_{f} m v^{2} \\
a_{0} & =c_{f} c_{r}\left(l_{f}+l_{r}\right)^{2}+\left(c_{r} l_{r}-c_{f} l_{f}\right) m v^{2} \\
a_{1} & =\left(c_{f}\left(J+l_{f}^{2} m\right)+c_{r}\left(J+l_{r}^{2} m\right)\right) v \\
a_{2} & =J m v^{2}
\end{aligned}
$$

The steady state gain of the nominal vehicle steering is

$$
K_{n}(v)=\left.\lim _{s \rightarrow 0} G(s)\right|_{\mu=\mu_{n}}
$$

at nominal friction coefficient $\mu=\mu_{n}$.

In this paper, the considered conventional car has a steer-by-wire actuator. Hence, the steering transfer function of the conventional car is

$$
G_{c o n v}=\frac{r}{\delta_{s}}=G G_{a}
$$

with $\delta_{s}$ denoting the steering wheel angle and $G_{o}$ being the actuator dynamics (see below).

A.1.1. Vehicle model data The nominal values of the variables for the linearized single track model assumed in this paper are $l_{f}=1.25 \mathrm{~m}$, $l_{r}=1.32 \mathrm{~m}, m=1296 \mathrm{~kg}, J=1750 \mathrm{~kg} \mathrm{~m}^{2}, c_{f 0}=$ $84243 \mathrm{~N} / \mathrm{rad}$ and $c_{r 0}=95707 \mathrm{~N} / \mathrm{rad}$. The nominal friction coefficient is $\mu_{n}=1$ which corresponds to dry road. 


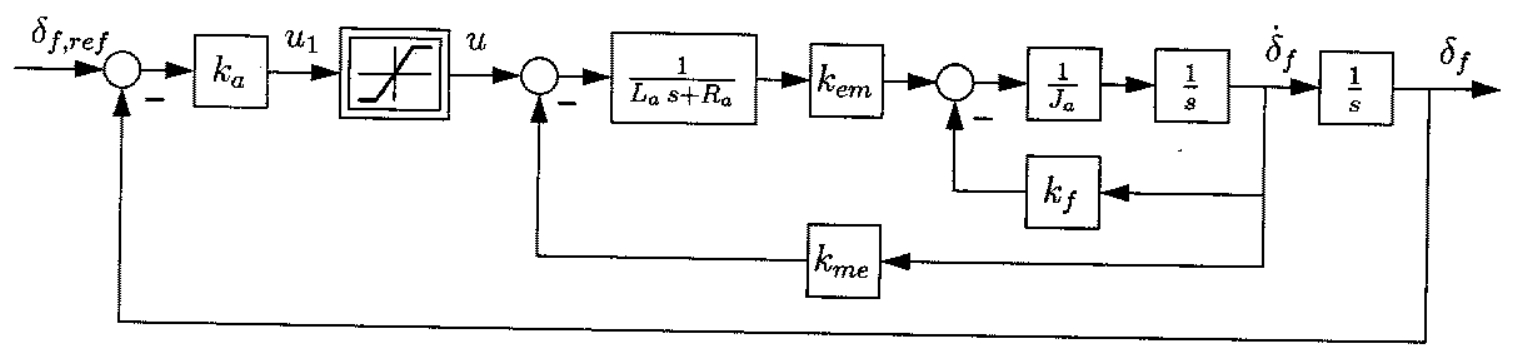

Fig. A.2. Actuator model

\section{A.2 Steering actuator model}

The steering actuator model (Ackermann et al., 2002) used in this paper is shown in Fig. A.2. The parameters of this model of an electric motor under position control are assumed to be $L=$ 0 (electric time constant neglected), $R=5 \Omega$, $J_{a}=0.004053 \mathrm{~kg} \mathrm{~m}{ }^{2}, k_{f}=0.01625, K_{a}=$ $22.22, k_{m e}=k_{e m}=0.9 \mathrm{Nm} / \mathrm{A}$. The electric voltage of the motor is assumed to be limited which is implemented in the model by a unity slope saturation between the signals $u_{1}$ and $u$. No assumptions on the saturation limits are necessary to be made in this paper. The transfer function of the linearized steering actuator model employed in sections $3-5$ is

$$
G_{a}=\frac{\delta_{f}}{\delta_{f r e f}}=\frac{\omega_{a}^{2}}{\omega_{a}^{2}+2 D_{a} \omega_{a} s+s^{2}}
$$

with $D_{a}=0.7$ and $\omega_{a}=2 \pi \cdot 5 \mathrm{~Hz}$ 